\title{
ON ALGEBRAS GENERATED BY INVERTIBLE OPERATORS
}

\author{
AVRAHAM FEINTUCH
}

\begin{abstract}
Let $T$ be an invertible operator such that the compact operators in $R(T)$ are weakly dense in it. Then $T^{-1} \in R(T)$. In particular, if $T$ is an essentially unitary $C_{0}$ operator, $T^{-1} \in R(T)$.
\end{abstract}

Let $T$ be an invertible operator on a separable complex Hilbert space $H$. If $H$ is finite dimensional, there exists a polynomial $p$ such that $T^{-1}=p(T)$. It is natural to consider the infinite dimensional analogue of this fact. Is $T^{-1}$ a limit of polynomials in $T$ in some oeprator topology? It is easy to see that in general the answer is negative even for the weak operator topology (example: the bilateral shift). It is obvious that for $T^{-1}$ to be a weak limit of polynomials in $T$, it is necessary for every invariant subspace of $T$ to be invariant under $T^{-1}$. It is not known if this is sufficient (see [1]).

Let Lat $T$ denote the lattice of closed invariant subspaces of $T$ and $R(T)$ the weak closure of the algebra of polynomials in $T$. Here we consider an operator $T$ for which the compact operators in $R(T)$ are weakly dense there. As shown by Nordgren in [2], essentially unitary $C_{0}$ contractions are such operators.

For a positive integer $n, \mathcal{H}^{(n)}$ will denote the usual direct sum of $n$ copies of $\mathcal{H}$ and $T^{(n)}$ will denote the direct sum of $n$ copies of $T$.

For an algebra $R, R^{(n)}=\left\{A^{(n)}: A \in R\right\}$. The following lemma is standard.

LEMMA 1. $B \in R(T)$ if and only if Lat $T^{(n)} \subseteq$ Lat $B^{(n)}$ for all positive integers $n$.

Lemma 2. Suppose $T$ is invertible and $\mathfrak{R}$ is a finite dimensional invariant subspace of $T$, then $\Re \in$ Lat $T^{-1}$.

Proof. If $\mathscr{T} \in$ Lat $T, T \mathfrak{T} \subseteq \mathfrak{T}$. Since $T$ is invertible, it preserves dimension. Thus $\operatorname{dim}(T \Re)=\operatorname{dim} \Re$. This implies $T \mathfrak{R}=\mathfrak{T}$.

THEOREM. Let $T$ be an invertible operator. If the compact operators in $R(T)$ are weakly dense there, then $T^{-1} \in R(T)$.

Proof. By Lemma 1, it suffices to show that Lat $T^{(n)} \subset$ Lat $T^{-1(n)}$. Since $T^{(n)}$ and $T$ satisfy the same hypotheses, the argument used for the ease $n=1$

Received by the editors April 20, 1976.

AMS (MOS) subject classifications (1970). Primary 47C05.

Key words and phrases. Invertible operators, weakly closed algebras, compact operators, invariant subspace. 
will be applicable to $n>1$. Thus, it is enough to show that Lat $T \subset$ Lat $T^{-1}$. In particular, it is enough to show that every cyclic subspace of $T$ is invariant under $T^{-1}$.

Denote the component of $\rho(T)$ containing zero by $\rho_{0}(T)$. Then for $\lambda \in$ $\rho_{0}(T)$, Lat $T^{-1}=\operatorname{Lat}(T-\lambda)^{-1}$.

For $x \in \mathcal{H}$, and $\lambda \in C$, it is clear that

$$
\bigvee_{n=0}^{\infty} T^{n} x=\bigvee_{n=0}^{\infty}(T-\lambda)^{n} x
$$

Let $\mathscr{R}=\bigvee_{n=0}^{\infty} T^{n} x \in$ Lat $T$. Let $\mathscr{T}$ be the span of all invariant subspaces of $T$ in $\Re$ which are in Lat $T^{-1}$.

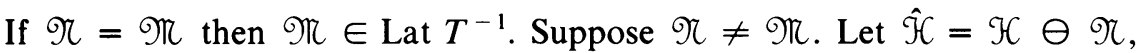
$\hat{\mathfrak{N}}=\mathfrak{N} \ominus \mathcal{X}$ and for an operator $A$ on $\mathcal{H}, \hat{A}$ the compression of $A$ to $\hat{\mathcal{H}}$. Since $\Re \in$ Lat $T \wedge$ Lat $T^{-1}, \hat{T}$ is invertible with inverse $\hat{T}^{-1}$. Thus we may as well assume $\Re=\{0\}$; i.e., $\Re$ contains no common invariant subspace of $T$ and $T^{-1}$.

Let $\left\{A_{K}\right\} \in R(T)$ be a net of compact operators which converges to $T$, and let $\lambda \in \rho_{0}(T)$ such that $|\lambda| \geqslant \delta>0$ for some $\delta$. Then

$$
\text { গ } \notin \operatorname{Lat}(T-\lambda)^{-1} \text {. }
$$

Since $T-\lambda$ is invertible, $(T-\lambda) \mathfrak{R}$ is a closed subspace of $\mathfrak{R}$ and since $\mathfrak{K}=\bigvee_{n=0}^{\infty}(T-\lambda)^{n} x$, $\Re \ominus(T-\lambda) \mathfrak{M}$ has dimension one. Thus on $\mathfrak{K}=$ $(T-\lambda) \mathfrak{R} \oplus \mathfrak{T}_{\lambda}$

$$
A_{K} \mid \Re=\left(\begin{array}{cc}
B_{K} & C_{K} \\
0 & \lambda_{K}
\end{array}\right) .
$$

Since $\Re_{\lambda}$ is one-dimensional,

$$
(T-\lambda) \mid \Re=\left(\begin{array}{cc}
T_{1} & T_{2} \\
0 & 0
\end{array}\right)
$$

Since $A_{K}-\lambda$ converges to $T-\lambda$, if follows that $\lambda_{K}$ converges to $\lambda$. Thus for $K$ sufficiently large, $\lambda_{K} \neq 0$. Note that $\lambda_{K} \in \sigma\left(A_{K} \mid \Re\right)$. Since $A_{K}$ is compact, $\lambda_{K}$ is an eigenvalue of $A_{K} \mid \Re$ and the corresponding eigenspace $\rho_{\lambda}$ is finite dimensional. Since $T$ commutes with $A_{K}$ on $\Re, \varrho_{\lambda} \in$ Lat $T \mid \mathscr{T}$ and thus $\varrho_{\lambda} \in$ Lat $T$. Since $\varrho_{\lambda}$ is finite dimensional, $\varrho_{\lambda} \in$ Lat $T^{-1}$ by Lemma 2 . This contradicts the fact that $\Re$ contains no common invariant subspace of $T$ and $T^{-1}$. The proof is complete.

Corollary. If $T$ is an essentially unitary $C_{0}$ contraction, $T^{-1} \in R(T)$.

REMARKS. The above theorem suggests the following two questions:

(1) Let $R$ be a commutative algebra generated by compact operators. Is $R$ inverse closed; i.e., for $T \in R$ invertible, is $T^{-1} \in R$ ? It is clear that the commutativity condition is essential. For consider the following example. Let $\mathcal{H}=l^{2}(-\infty ; \infty)$ with the usual basis $\left\{e_{n}\right\}_{n=-\infty}^{\infty}$. Define $F_{n}$ by

$$
F_{n} e_{j}= \begin{cases}e_{j+1}, & j=n, \\ 0, & j \neq n,\end{cases}
$$


Then $F_{n}$ is of rank one. Let $R$ be the weakly closed algebra generated by $\left\{F_{n}\right\}$. Then $R$ contains the bilateral shift $W$. Also $l^{2}(0 ; \infty) \in$ Lat $R$. Thus $W^{-1} \notin R$.

(2) Suppose $T$ is invertible and $R(T)$ contains an injective compact operator. Is $T^{-1} \in R(T)$ ?

\section{BIBLIOGRAPHY}

1. A Feintuch, On invertible operators and invariant subspaces, Proc. Amer. Math. Soc. 43 (1974), 123-126. MR 48 \#9415.

2. E. A. Nordgren, Compact operators in the algebra generated by essentially unitary $C_{0}$ operators, Proc. Amer. Math. Soc. 51 (1975), 159-162. MR 51 \#6451.

3. D. Sarason, The $H^{p}$ spaces of an annulus, Mem. Amer. Math. Soc. No. 56 (1965). MR 32 \#6256.

Department of Mathematics, Ben Gurion University of the Negev, Beer Sheva, Israel 